\title{
A Staged Finite-Time Control Strategy for Formation of Underactuated Unmanned Surface Vehicles
}

\author{
Hui Ye $\mathbb{D}^{-}$, Xiaofei Yang $\left(\mathbb{D}\right.$, Chunxiao Ge $\mathbb{D}^{\text {D, }}$, and Zhaoping Du \\ School of Electronics and Information, Jiangsu University of Science and Technology, Zhenjiang 212003, China \\ Correspondence should be addressed to Hui Ye; yehuicc@just.edu.cn
}

Received 1 December 2020; Revised 16 December 2020; Accepted 30 December 2020; Published 13 January 2021

Academic Editor: Rui Wang

Copyright (C) 2021 Hui Ye et al. This is an open access article distributed under the Creative Commons Attribution License, which permits unrestricted use, distribution, and reproduction in any medium, provided the original work is properly cited.

\begin{abstract}
The formation control issue for a group of underactuated unmanned surface vehicles (USVs) is discussed in the paper, and a staged finite-time control strategy for the USVs is proposed. Firstly, we try to steer each USV to its own starting point in the formation for a limited time, under the initial condition that each of these vehicles is parked at random. To deal with the nonholonomic behavior of the system, the dynamics of the USV is transformed into cascade systems. Then, the finite-time controller is designed for each vehicle based on homogeneity theory. After each USV reaches its own starting point with desired orientation, the model of the vehicle is decomposed into two subsystems under the Serret-Frenet frame. In order to maintain the formation pattern, two finite-time distributed controllers are developed for the surge subsystem and the yaw subsystem, respectively. The settling time for the staged control strategy is limited. Numerical simulations are carried out to illustrate the effectiveness of the proposed formation control strategy.
\end{abstract}

\section{Introduction}

Over the last decade, a great attention has been paid by scholars on unmanned surface vehicles (USVs), which can be used to perform various tasks, such as marine surveillance, oil spill collection, environmental monitoring, bathymetric mapping, and marine floor investigation [1]. These tasks are usually complexity and onerous, which are difficult to do by a single vehicle. Multivehicle cooperation is the inevitable choice, which can not only accomplish the tasks efficiently but also ensure personnel safety, reduce system cost, and provide redundancy against individual failure. Typical cooperative control issues of multiagent systems include consensus, distributed tracking, formation control, containment control, swarming, and aggregation. In this paper, the formation control of multiple USVs is considered.

There are two main difficulties in the formation control problem of underactuated USVs, one of which exists in the motion control of each single vehicle. Most existing USVs are typical underactuated systems, whose number of actuators is fewer than their degrees of freedom (DOF). The trajectory tracking control of the underactuated USV is studied in $[2,3]$, and the virtual target is adopted to generate the reference trajectory. The path following of the underactuated USV is usually addressed by introducing the SerretFrenet frame and global coordinate transformation $[4,5]$. Then, the underactuated problem is converted to a virtual fully actuated problem by designing the virtual control law. For the stabilization of the underactuated USVs, nonsmooth or time-varying cascaded design is usually proposed due to the nonholonomic constraints [6]. Moreover, finite-time controllers are developed in $[7,8]$ via adding one power integrator technique and terminal sliding mode method, respectively.

Another difficulty is to maintain formation pattern of multiple USVs, which will encounter issues of communication and coordination. From a review of the literature, there are mainly four methods used for the formation control of multiple vehicles, including virtual structure method [9], graph theory method [10], behavioral based approach [11], and leader-follower strategy [12, 13]. Among these methods, the leader-follower strategy is most preferred in practice for the formation of USVs because of its high 
reliability and easily implementation [14]. A robust adaptive formation controller is proposed in [15] for the formation of USVs with uncertainties and ocean disturbances, where neural networks and dynamic surface control technique are used to capture them. In [16], a parameter estimation- and upper bound estimation-based sliding mode control scheme is presented to deal with the model uncertainties and environmental disturbances. The control constraint issues in the formation of underactuated USV s are also considered by many scholars. In [17], a formation tracking controller is developed by using generalized saturation functions to reduce the risk of actuator saturation. The receding horizon control algorithm is adopted in [18] for the formation tracking of a feet of underactuated autonomous underwater vehicles (AUVs) with constrained inputs. In [19], auxiliary dynamic systems and disturbance observers are designed to deal with input saturations and disturbances respectively, and a robust controller is developed for time-varying formation control of underactuated AUVs. The fault tolerant control problem in the leader-follower formation of USVs is considered in [20], while both parametric and nonparametric system uncertainties are discussed.

Another popular method for USV formation control is based on the graph theory, which is proposed to settle the consensus problem of multiagents. The literature [21] considers the cooperative control of a group of underactuated USVs using the relative information between neighbor's information with the aid of results from graph theory and Lyapunov techniques. In [22], a distributed maneuvering controller of multiple USVs is designed in the presence of unknown state constraints. An RNN-based command governor is provided between the kinematic controller and the kinetics controller. A cooperative path maneuvering controller is designed based on a line of sight guidance scheme in [23], where the modular adaptive control method is employed to identify uncertain kinematics and unknown kinetics of each vehicle.

Currently, finite-time formation control of multiple USVs become an active research area, since a lot of works have been carried out in both the finite-time control of single USV [24-26] and the finite-time consensus of multiagents [27-29]. In [30], the finite-time formation control of underactuated USVs is investigated with consideration of model uncertainty and environmental disturbance. The reference trajectory of each USV is given first according to the desired geometric pattern. Then, a nonlinear controller with fractional power feedback is designed for each USV, to make sure each vehicle follows its own trajectory in finite time. In [31], the terminal sliding mode approach is used to deal with the finite-time formation of underactuated ships. Specifically, a generalized error which contains topology information of communication is defined so that the method based on graph theory can be used for the nonlinear system.

Motivated by the aforementioned works, the finite-time control for single USV and the finite-time cooperative control for multiple USVs are integrated is this paper. A staged finite-time control scheme is proposed for the formation of multiple underactuated USVs. Our major contributions are listed as follows:
(1) To deal with the underactuated nature and achieve the goal of finite-time convergence, we mainly separate the formation control process into two stages. In the first stage, we try to steer each USV to its own starting point in the formation configuration for a limited time. In the second stage, the formation pattern is maintained by using two finite-time cooperative controllers. As a consequence, the resulting control scheme can guarantee finite-time convergence.

(2) In the first stage, the positioning problem of every single underactuated USV is regard as a finite-time stability problem of the homogeneous system. Homogeneous theory is applied in the control design, to make each vehicle get to its own position in the formation pattern.

(3) In the second stage, the model of each USV can be decoupled into two subsystems with a series of integrators: the surge subsystem and the yaw subsystem. Taking communication link topology into account, two finite-time cooperative controllers are designed based on graph theory to maintain the formation pattern.

The remainders of the paper are organized as follows. In Section 2, preliminaries and problem formulation are introduced. Section 3 presents the main results on the staged control algorithm design, where finite-time control for single USV is designed in Section 3.1 and finite-time cooperative control for formation maintenance is given in Section 3.2. In order to illustrate the effectiveness of the proposed control laws, numerical simulation results are presented in Section 4. Finally, some conclusion remarks are drawn in Section 5.

\section{Preliminary}

In this section, theories of finite-time stability, homogeneity, and algebraic graph are introduced briefly, and some lemmas are given. Then, the kinematics and dynamics of the underactuated USV are provided, and the task of formation control for multiple USVs is summarized.

2.1. Finite-Time Stability and Homogeneity. Both asymptotic stability and finite-time convergence of the closed-loop system are required in our design, which leads to the conception of the finite-time stability.

Definition 1. Consider the system

$$
\begin{aligned}
\dot{x} & =\mathbf{f}(\mathbf{x}), \\
\mathbf{f}(0) & =0, \quad \mathbf{x} \in \mathbb{R}^{n},
\end{aligned}
$$

where $\mathbf{f}: \mathbb{R}^{n} \longrightarrow \mathbb{R}^{n}$ is continuous. Assume that, for every initial condition $\mathbf{x}(0)=\mathbf{x}_{0} \in \mathbb{R}^{n}$, system (1) possesses a unique solution $\mathbf{x}(t)=\xi\left(t, \mathbf{x}_{0}\right)$ in forward time which is defined on $[0, \infty)$. The origin is said to be a finite-time stable equilibrium of (1) if there exists an open neighborhood $\mathcal{U}$ of 
the origin and a function $t_{f}: \mathscr{U} \longrightarrow[0, \infty)$ called the settling time function such that the following statements hold:

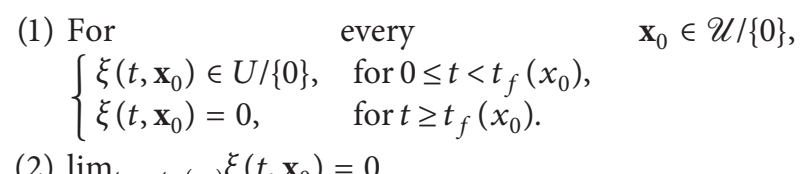

(2) $\lim _{t \longrightarrow t_{f}\left(\mathbf{x}_{0}\right)} \xi\left(t, \mathbf{x}_{0}\right)=0$.

The origin is said to be a globally finite-time stable equilibrium if it is a finite-time stable equilibrium and $\mathcal{U}=\mathbb{R}^{n}$.

In general, there are three techniques which can be used to achieve finite-time stability for the feedback stabilization, including homogeneous theory, terminal sliding mode method, and adding one power integrator approach [32]. In this paper, nonsmooth feedback finite-time controllers are designed with the help of the homogeneous system.

Definition 2 (see [32]). Consider the dilation of the form $\delta_{\varepsilon}^{\left(\gamma_{1}, \ldots, \gamma_{n}\right)}\left(x_{1}, \ldots, x_{n}\right)=\left(\varepsilon^{\gamma_{1}} x_{1}, \ldots, \varepsilon^{\gamma_{n}} x_{n}\right)$, where $x_{1}, \ldots, x_{n}$ are suitable coordinates on $\mathbb{R}^{n}$ and $\gamma_{1}, \ldots, \gamma_{n}$ are positive real numbers. A vector field is homogeneous of degree $k$ if and only if the $i^{\text {th }}$ component $f_{i}$ is homogeneous of degree $k+\gamma_{i}$. System (1) is said to be a homogeneous system if its vector field $\mathbf{f}$ is homogeneous.

The connection between the finite-time stability and the homogeneous system is concluded in the following two lemmas.

Lemma 1 (see [32]). For system (1), suppose there exists an open neighborhood $\mathcal{U}$ of the origin, a $\mathscr{C}^{1}$ positive definite function $V(\mathbf{x})$, and real number $\varepsilon>0$ and $0<\alpha<1$, such that $\dot{V}(\mathbf{x})+\varepsilon V^{\alpha}(\mathbf{x})$ is negative semidefinite on $\mathcal{U}$. Then, the origin is a finite-time stable equilibrium of the system.

Lemma 2 (see [32]). Suppose that system (1) is homogeneous of degree $k$ with respect to $\left(\gamma_{1}, \ldots, \gamma_{n}\right)$, the origin is a finitetime stable equilibrium of (1) if and only if the origin is an asymptotically stable equilibrium of (1) and $k<0$.

Consider the following chain of integrators:

$$
\begin{array}{cc}
\dot{x}_{1}=x_{2}, & x_{1}\left(t_{0}\right)=x_{10}, \\
\dot{x}_{2}=x_{3}, & x_{2}\left(t_{0}\right)=x_{20}, \\
\vdots \quad \vdots & \\
\dot{x}_{n}=u, & x_{n}\left(t_{0}\right)=x_{n 0} .
\end{array}
$$

System (2) is finite-time convergent to the origin if the control input is designed as

$$
u=-k_{1} x_{1}^{\alpha_{1}}-k_{2} x_{2}^{\alpha_{2}}-\cdots-k_{n} x_{n}^{\alpha_{n}},
$$

where the exponents $\alpha_{i} \in(0,1), i=1, \ldots, n-1$, are selected satisfying

$$
\begin{aligned}
\alpha_{i} & =\frac{\alpha_{i+1} \alpha_{i+2}}{\left(2 \alpha_{i+2}-\alpha_{i+1}\right)}, \\
\alpha_{n+1} & =1, \\
\alpha_{n} & =\alpha, \quad 0<\alpha<1 .
\end{aligned}
$$

The item $x_{i}^{\alpha_{i}}$ is defined as $\left|x_{i}\right|^{\alpha_{i}} \operatorname{sgn}\left(x_{i}\right)$, where $\operatorname{sgn}(\cdot)$ is the sign function. The gains $k_{i}, i=1, \ldots, n$, are selected such that the following matrix is Huiwitz:

$$
\mathbf{A}_{x}=\left(\begin{array}{ccccc}
0 & 1 & 0 & \ldots & 0 \\
0 & 0 & 1 & \ldots & 0 \\
\vdots & \vdots & \vdots & \ddots & \vdots \\
0 & 0 & 0 & \ldots & 1 \\
-k_{1} & -k_{2} & -k_{3} & \ldots & -k_{n}
\end{array}\right)
$$

Define the Lyapunov function candidate $V(\zeta)=\zeta^{T} \mathbf{P} \zeta$ with $\zeta=\left(x_{1}^{\alpha_{1}}, x_{2}^{\alpha_{2}}, \ldots, x_{n}^{\alpha_{n}}\right)^{T}$. $\mathbf{P}$ and $\mathbf{Q}$ are symmetric positive definite matrices satisfying

$$
\mathbf{P} \mathbf{A}_{\mathbf{x}}+\mathbf{A}_{x}^{T} \mathbf{P}=-\mathbf{Q} .
$$

Lemma 3 (see [33]). Consider system (2) together with (3). The state $\mathbf{x}=\left(x_{1}, x_{2}, \ldots, x_{n}\right)^{T}$ converges to the origin for a finite time. Moreover, the upper bound of the settling time can be estimated as

$$
t_{f}\left(\mathbf{x}_{0}\right) \leq \frac{\alpha V\left(\zeta_{0}\right)^{(1-\alpha / \alpha)}}{(1-\alpha) \rho}
$$

for all $\mathbf{x}_{0}=\left(x_{10}, x_{20}, \ldots, x_{n 0}\right)^{T}$ in some open neighborhood $\mathcal{U}$ of the origin, where $\rho=\lambda_{\text {min }}(\mathbf{Q}) / \lambda_{\text {max }}(\mathbf{P}) . \lambda_{\min }(\mathbf{Q})>0$ is the minimum eigenvalue of the matrix $\mathbf{Q}$, and $\lambda_{\text {max }}(\mathbf{P})>0$ is the maximum eigenvalue of the matrix $\mathbf{P}$.

2.2. Graph Theory. The communication links topology among the group of NSVs can be represented by a graph $\mathscr{G}=\{\mathscr{V}, \mathscr{E}, \mathscr{A}\}$, where $\mathscr{V}=\left\{v_{1}, v_{2}, \ldots, v_{m}\right\}$ is the set of vehicles, $\mathscr{E}$ represents the set of links between the vehicles (also called edge set), and $\mathscr{A}$ is the weighted coefficients of edge set $\mathscr{E}$. In the directed graph, $(i, j) \in \mathscr{E}$ represents the $j^{\text {th }}$ vehicle can communicate with $i^{\text {th }}$ vehicle, but not conversely. The $i^{\text {th }}$ vehicle is called the parent node, and the $j^{\text {th }}$ vehicle is also called the child node of the $i^{\text {th }}$ vehicle, and they are neighbors. In the undirected graph, $(i, j) \in \mathscr{E}$ denotes the information can be exchanged between the two vehicles. All vehicles are connected with a single undirected path. However, in the directed graph, each vehicle has only one parent node, except for one vehicle, which is called the root. If $m$ vehicles are in the scenario, then the associated adjacency matrix of $\mathscr{G}$ can be defined as $\mathbf{A}=\left\{a_{i j}\right\}_{m \times m} \in \mathbb{R}^{m \times m}$. $a_{i j}>0$ if and only if there is a link from $j^{\text {th }}$ vehicle to $i^{\text {th }}$ vehicle; otherwise, $a_{i j}=0$. The Laplacian matrix $\mathbf{L}=\left\{l_{i j}\right\}_{m \times m} \in \mathbb{R}^{m \times m}$ associated with the matrix $\mathbf{A}$ is defined by $l_{i i}=\sum_{j=1}^{m} a_{i j}$ and $l_{i j}=-a_{i j}$, when $i \neq j$. The Laplacian matrix can be written as $\mathbf{L}=\mathbf{D}-\mathbf{A}$, where $\mathbf{D}$ is the degree matrix [34].

Consider a virtual leader $v_{0}$ moving with the group centroid. Define the augmented graph as $\overline{\mathscr{G}}=\{\overline{\mathscr{V}}, \overline{\mathscr{E}}, \overline{\mathscr{A}}\}$, where $\overline{\mathscr{V}}=\left\{v_{0}, v_{1}, \ldots, v_{m}\right\}$ and $\overline{\mathscr{E}}=\subseteq \mathscr{V} \times \mathscr{V}$ are the new node set and edge set, respectively. Diagonal matrix $\mathbf{B}=$ $\operatorname{diag}\left\{a_{10}, a_{20}, \ldots, a_{m 0}\right\}$ is called the leader adjacency matrix, 
where $a_{i 0}>0$ if follower $i$ is connected to the leader; otherwise, $a_{i 0}=0$. In this work, the communication topology verifies the following assumption.

Assumption 1. The graph $\mathscr{G}$ associated with the follower is undirected, and in the graph $\overline{\mathscr{G}}$ the leader has directed path to all followers.

Lemma 4 (see [35]). Under Assumption 1, L + B is a symmetric positive definite matrix.

2.3. Mathematical Model of Underactuated USV. Two reference coordinate systems are defined to describe the model of the USV, where $\mathrm{X}_{\mathrm{E}} \mathrm{O}_{\mathrm{E}} \mathrm{Y}_{\mathrm{E}}$ is the earth-fixed inertial frame and $\mathrm{X}_{\mathrm{b}} \mathrm{O}_{\mathrm{b}} \mathrm{Y}_{\mathrm{b}}$ is the body-fixed frame, as shown in Figure 1 . The model state of the USV in horizontal plane is given by $(x, y, \psi, u, v, r)$, where $x, y$, and $\psi$ denote the position and orientation of the vehicle in the earth-fixed frame, $u, v$, and $r$ denote the surge, sway, and yaw velocity of the vehicle in the body-fixed frame, respectively.

Based on the above definition, the kinematic model of the USV can be described as

$$
\begin{aligned}
& \dot{x}=u \cos \psi-v \sin \psi, \\
& \dot{y}=u \sin \psi+v \cos \psi, \\
& \dot{\psi}=r .
\end{aligned}
$$

And the dynamic model of the USV can be given by

$$
\begin{aligned}
m_{u} \dot{u}-m_{v} v r+d_{u} u & =\tau_{u}, \\
m_{v} \dot{v}+m_{u} u r+d_{v} v & =0, \\
m_{r} \dot{r}-m_{u v} u v+d_{r} r & =\tau_{r},
\end{aligned}
$$

where $d_{u}, d_{v}$, and $d_{r}$ are the hydrodynamic damping of the vehicle and $m_{u}, m_{v}$, and $m_{r}$ are the inertia of the vehicle including added masses in surge, sway, and yaw. Besides, $m_{u v}$ is the hydrodynamic added mass and $\tau_{u}$ and $\tau_{r}$ are the surge force and yaw moment, respectively.

It can be seen from (9) that the USV can only get the surge force and yaw moment from the thrusters and the

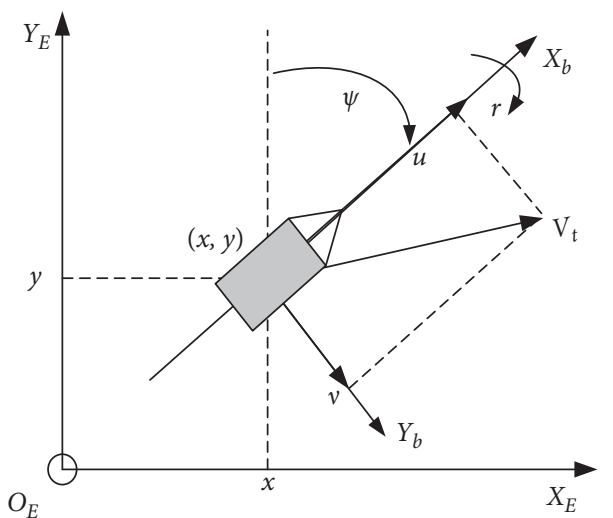

FIgURE 1: The earth-fixed inertial and the body-fixed frame.

rudder in the stern, and there is no sway force from the vessel thruster, so the vehicle is a typical underactuated system.

2.4. Formation of Multiple USVs. Consider a group of $m$ USVs, whose mathematical models are expressed as (8) and (9). For clarity, the subscript " $i$ " $(i=1, \ldots, m)$ is used to distinguish one from another. Such as, the state of the USV $i$ is denoted by $\left(x_{i}, y_{i}, \psi_{i}, u_{i}, v_{i}, r_{i}\right)$. Assume that all the USVs are scattered randomly in the water at any initial states. The goal of this work is to steer these vehicles to achieve the prescribed formation pattern along a given straight line or arc path with a desired speed. Moreover, the settling time is required to be finite. Without loss of generality, the earthfixed frame is defined with its origin located on the center of the formation at starting point. The $\mathrm{X}_{\mathrm{E}}$-axis coincides with the tangent of path with the positive axis pointing to the motion direction of the group, as shown in Figure 2.

A feasible desired trajectory can be described by the states $\left(x_{0}, y_{0}, \psi_{0}, u_{0}, v_{0}, r_{0}\right)$ of the group centroid which is moving along the path. The object of the control system can be expressed as follows.

In the first stage:

$$
\left\{\begin{array}{l}
\lim _{t \longrightarrow t_{f 1}} x_{i}(t)-x_{0}\left(t_{f 1}\right)=\Delta x_{i}, \\
\lim _{t \longrightarrow t_{f 1}} y_{i}(t)-y_{0}\left(t_{f 1}\right)=\Delta y_{i}, \\
\lim _{t \longrightarrow t_{f 1}} \psi_{i}(t)-\psi_{0}\left(t_{f 1}\right)=\Delta \psi_{i},
\end{array},\left\{\begin{array}{l}
\lim _{t \longrightarrow t_{f 1}} u_{i}(t)=0, \\
\lim _{t \longrightarrow t_{f 1}} v_{i}(t)=0, \quad \text { for } i=1, \ldots, m, \\
\lim _{t \longrightarrow t_{f 1}} r_{i}(t)=0 .
\end{array}\right.\right.
$$

In the second stage:

$$
\left\{\begin{array}{l}
\lim _{t \rightarrow t_{f 2}}\left(s_{i}(t)-s_{0}(t)\right)=\Delta s_{i}, \\
\lim _{t \longrightarrow t_{f 2}}\left(d_{i}(t)-d_{0}(t)\right)=\Delta d_{i},
\end{array} \quad \text { for } i=1, \ldots, m,\right.
$$

where $\left(\Delta x_{i}, \Delta y_{i}, \Delta \psi_{i}\right)$ are the relative position and orientation of USV $i$ to the group centroid, $\left(s_{i}, d_{i}\right)$ are longitudinal and transversal displacements of USV $i$ with respect to the path, and $\left(\Delta s_{i}, \Delta d_{i}\right)$ are the longitudinal and transversal distances between USV $i$ and the group centroid. 


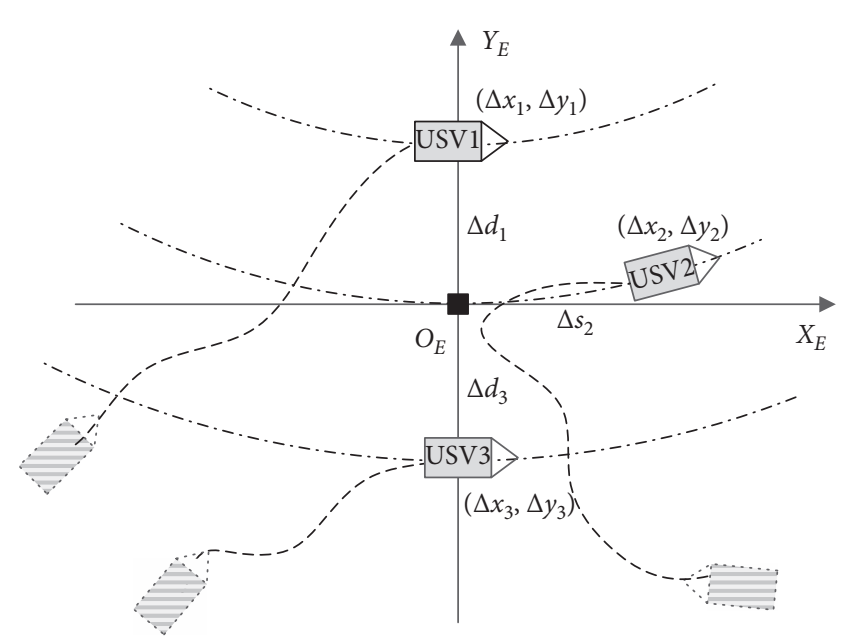

FIGURE 2: The reference frame and the formation pattern of the NSVs.

\section{Staged Control Design for the Formation of USVs}

For formation control of the USVs, vehicles should be forced to achieve the desired formation firstly and then maintain the geometric configuration. Consequently, the proposed control strategy includes two stages. In the first stage, each vehicle is steered to its own position in the formation pattern within a finite time. After that, the dynamics of the underactuated USV can be transformed into two integrator systems. In the second stage, two finite-time cooperative controllers are employed to maintain the formation shape and guarantee the stabilities of the systems.

3.1. Finite-Time Control for Single USV. In this stage, each vehicle is controlled separately to reach its initial position in the formation. We hope that this process could be completed in a limited time. For the underactuated USV $i$, its desired pose vector to the group centroid is $\left(\Delta x_{i}, \Delta y_{i}, \Delta \psi_{i}\right)$. We can transform the set point tracking problem of USV $i$ to the stabilization problem by defining the following new state variables:

$$
\left\{\begin{array}{l}
\bar{x}_{i}=x_{i}-\Delta x_{i} \\
\bar{y}_{i}=y_{i}-\Delta y_{i} \\
\bar{\psi}_{i}=\psi_{i}-\Delta \psi_{i}
\end{array}\right.
$$

Then, the kinematic equation of USV $i$ can be rewritten as

$$
\left\{\begin{array}{l}
\dot{\bar{x}}_{i}=u_{i} \cos \bar{\psi}_{i}-v_{i} \sin \bar{\psi}_{i} \\
\dot{\bar{y}}_{i}=u_{i} \sin \bar{\psi}_{i}+v_{i} \cos \bar{\psi}_{i} \\
\dot{\bar{\psi}}_{i}=r_{i} .
\end{array}\right.
$$

Since the mathematical model of the underactuated USV is a typical nonholonomic system, the following global coordinate transformation is employed before control design [36]:

$$
\left\{\begin{array}{l}
z_{1 i}=\bar{x}_{i} \cos \bar{\psi}_{i}+\bar{y}_{i} \sin \bar{\psi}_{i}, \\
z_{2 i}=-\bar{x}_{i} \sin \bar{\psi}_{i}+\bar{y}_{i} \cos \bar{\psi}_{i}+\frac{m_{v}}{d_{v}} v_{i}, \\
z_{3 i}=\bar{\psi}_{i}, \\
z_{4 i}=-\frac{m_{u}}{d_{v}} u_{i}-z_{1 i}, \\
z_{5 i}=v_{i}, \\
z_{6 i}=r_{i}, \\
\tau_{1 i}=\left(\frac{d_{u}}{d_{v}}-1\right) u_{i}-z_{2 i} z_{6 i}-\frac{\tau_{u i}}{d_{v}} \\
\tau_{2 i}=\frac{m_{u v}}{m_{r}} u_{i} v_{i}-\frac{d_{r}}{m_{r}} r_{i}+\frac{\tau_{r i}}{m_{r}},
\end{array}\right.
$$

where $\left(\tau_{1 i}, \tau_{2 i}\right)$ represents the new input transformation pattern. Thus, system (13) together with (9) can be transformed into

$$
\begin{aligned}
& \left\{\begin{array}{l}
\dot{z}_{2 i}=z_{4 i} z_{6 i}, \\
\dot{z}_{3 i}=z_{6 i}, \\
\dot{z}_{4 i}=\tau_{1 i}, \\
\dot{z}_{6 i}=\tau_{2 i}
\end{array}\right. \\
& \left\{\begin{array}{l}
\dot{z}_{1 i}=-\frac{d_{v}}{m_{u}} z_{1 i}-\frac{d_{v}}{m_{u}} z_{4 i}+z_{2 i} z_{6 i}-\frac{m_{v}}{d_{v}} z_{5 i} z_{6 i} \\
\dot{z}_{5 i}=-\frac{d_{v}}{m_{v}} z_{5 i}+\frac{d_{v}}{m_{v}} z_{6 i}\left(z_{1 i}+z_{4 i}\right) .
\end{array}\right.
\end{aligned}
$$

Remark 1 (see [36]). The system stabilization problem in (13) and (9) is equal to systems (15) and (16). If system (16) is global asymptotic convergent within finite time, the overall system of (15) and (16) is global asymptotic convergent.

Remark 2. As $\left(\tau_{u i}, \tau_{r i}\right)$ and $\left(\tau_{1 i}, \tau_{2 i}\right)$ can be converted to each other, just $\left(\tau_{1 i}, \tau_{2 i}\right)$ need to be designed to make variables $z_{1 i}, z_{2 i}, z_{3 i}, z_{4 i}, z_{5 i}$, and $z_{6 i}$ asymptotically converge to zero, which means that the tuple $\left(x_{i}, y_{i}, \psi_{i}, u_{i}, v_{i}, r_{i}\right)$ converges to $\left(\Delta x_{i}, \Delta y_{i}, \Delta \psi_{i}, 0,0,0\right)$. That is to say, the USV has arrived to its initial place in the formation pattern with desired orientation.

In view of the above remarks, our objective has shifted from set point tracking for each USV to simply stabilizing system (15). Note that this system does not satisfy Brockett's necessary condition. Hence, we divide system (15) into two cascade subsystems and try to provide a discontinuous 
control law. Suppose $z_{6 i}$ is not zero at the initial time, such as $z_{6 i}(0) \neq 0$. The first cascade subsystem is given as the follows:

$$
\left\{\begin{array}{l}
\dot{z}_{2 i}=z_{4 i}^{\prime}, \\
\dot{z}_{4 i}^{\prime}=\tau_{1 i}^{\prime},
\end{array}\right.
$$

where $z_{4 i}^{\prime}=z_{6 i}(0) z_{4 i}$ and $\tau_{1 i}^{\prime}=z_{6 i}(0) \tau_{1 i}$. The finite-time control law is designed as

$$
\begin{aligned}
& \tau_{1 i}^{\prime}=-k_{1 i} z_{2 i}^{\alpha_{1}}-k_{2 i} z_{4 i}^{\alpha_{2}}, \\
& \tau_{2 i}=0, \quad \text { for } t \in\left[0, t_{1 i}\right],
\end{aligned}
$$

where the exponents $\alpha_{1}, \alpha_{2}$ are selected as $\alpha_{1}=\alpha_{2} /\left(2-\alpha_{2}\right), 0<\alpha_{2}<1$. The gains $k_{1 i}$ and $k_{2 i}$ are selected to satisfy

$$
\mathbf{P}_{1 i}\left(\begin{array}{rr}
0 & 1 \\
-k_{1 i} & -k_{2 i}
\end{array}\right)+\left(\begin{array}{rr}
0 & 1 \\
-k_{1 i} & -k_{2 i}
\end{array}\right)^{T} \mathbf{P}_{1 i}=-\mathbf{Q}_{1 i}
$$

Thus, $t_{1 i}$ is the settling time which can be calculated by

$$
t_{1 i}=\frac{\alpha_{2}\left(\zeta_{1 i}^{T}(0) \mathbf{P}_{1 i} \zeta_{1 i}(0)\right)^{\left(1-\alpha_{2}\right) / \alpha_{2}}}{\left(1-\alpha_{2}\right) \rho_{1 i}}
$$

where $\zeta_{1 i}(0)=\left(z_{2 i}(0), z_{4 i}(0)\right)^{T}$ and $\rho_{1 i}=\lambda_{\min }\left(\mathbf{Q}_{1 i}\right) /$ $\lambda_{\max }\left(\mathbf{P}_{1 i}\right)$. According to Lemma 3 , the feedback control law (18) presents a homogeneous closed-loop system with negative degree of homogeneity. Thus, the states $z_{2 i}$ and $z_{4 i}$ of system (17) reach to zero in finite time; then, $\tau_{2 i}$ would not affect system (17) any more. Moreover, $\tau_{2 i}$ can be designed independently for the second cascade system:

$$
\left\{\begin{array}{l}
\dot{z}_{3 i}=z_{6 i} \\
\dot{z}_{6 i}=\tau_{2 i} .
\end{array}\right.
$$

After time $t_{1 i}$, we have

$$
\begin{aligned}
& \tau_{1 i}^{\prime}=0, \\
& \tau_{2 i}=-k_{3 i} z_{3 i}^{\alpha_{3}}-k_{4 i} z_{6 i}^{\alpha_{4}}, \quad \text { for } t \in\left(t_{1 i}, t_{1 i}+t_{2 i}\right],
\end{aligned}
$$

where the exponents $\alpha_{3}$ and $\alpha_{4}$ are selected as $\alpha_{3}=\alpha_{4} /$ $\left(2-\alpha_{4}\right), 0<\alpha_{4}<1$. The gains $k_{3 i}$ and $k_{4 i}$ are selected to satisfy

$$
\mathbf{P}_{2 i}\left(\begin{array}{rr}
0 & 1 \\
-k_{3 i} & -k_{4 i}
\end{array}\right)+\left(\begin{array}{rr}
0 & 1 \\
-k_{3 i} & -k_{4 i}
\end{array}\right)^{T} \mathbf{P}_{2 i}=-\mathbf{Q}_{2 i} .
$$

Thus, $t_{2 i}$ is the settling time which can be calculated by

$$
t_{2 i}=\frac{\alpha_{4}\left(\zeta_{2 i}^{T}\left(t_{1 i}\right) \mathbf{P}_{2 i} \zeta_{2 i}\left(t_{1 i}\right)\right)^{\left(1-\alpha_{4}\right) / \alpha_{4}}}{\left(1-\alpha_{4}\right) \rho_{2 i}},
$$

where $\zeta_{1 i}\left(t_{1 i}\right)=\left(z_{3 i}\left(t_{1 i}\right), z_{6 i}\left(t_{1 i}\right)\right)^{T}$ and $\rho_{2 i}=\lambda_{\min }\left(\mathbf{Q}_{2 i}\right) / \lambda_{\max }$ $\left(\mathbf{P}_{2 i}\right)$. By using Lemma 3 again, the control law (22) makes the states $z_{3 i}$ and $z_{6 i}$ of system (21) converge to zero in finite time.

After that the states of (15) converge to zero, system (16) is also asymptotically stabilized according to Remark 1. Actually, system (16) is simplified to first-order systems, whose settling time periods are $3 m_{u} / d_{v}$ and $3 m_{v} / d_{v}$. We let

$$
t_{3 i}=\max \left\{\frac{3 m_{u}}{d_{v}}, \frac{3 m_{v}}{d_{v}}\right\} .
$$

So, the final convergence time of all USVs is calculated as

$$
t_{f 1}=\max _{i}\left\{t_{1 i}+t_{2 i}+t_{3 i}\right\}
$$

That is to say, all USV san arrive to their positions in the formation pattern after time $t_{f 1}$.

3.2. Cooperative Control for Formation Maintenance. The goal of the second stage is to maintain the formation pattern by means of finite-time distributed control. It is assumed that only local distributed state information would be utilized by each vehicle to make its own surge velocity and yaw angular consistent with others. Consider a virtual leader moving with the group centroid, labeled 0 . The communication graph $\overline{\mathscr{G}}$ among the $n$ vehicles and the virtual leader satisfies Assumption 1.

After the first stage, the group of USV s has come into a prespecified geometric pattern with desired yaw angular. A reference path $\Gamma_{0}$ is previously specified for the centroid of the group which must be followed by the group of the USVs, as shown in Figure 3. The kinematic model of each vehicle can be rewritten according to the Serret-Frenet frame. Suppose that there is a virtual S-F frame $\mathrm{X}_{\mathrm{t}} \mathrm{O}_{0} \mathrm{X}_{\mathrm{n}}$ moving along the path $\Gamma_{0}$ for the virtual vehicle 0 . The kinematics of vehicle $i$ expressed in new state coordinates is [37]

$$
\begin{aligned}
& \dot{d}_{i}=u_{i} \sin \varphi_{i}+v_{i} \cos \varphi_{i}, \\
& \dot{\varphi}_{i}=r_{i}-c \dot{s}_{i}, \\
& \dot{s}_{i}=\frac{1}{1-c d_{i}}\left(u_{i} \cos \varphi_{i}-v_{i} \sin \varphi_{i}\right),
\end{aligned}
$$

where $\varphi_{i}=\psi_{i}-\Delta \psi_{i}-\psi_{0}$ and $c$ is the curvature of the path which is constant for the straight line or arc. Since each vehicle is on its own path and tangent to it at the initial moment, we assume that $d_{i} \approx \Delta d_{i}, v_{i} \approx 0$ and $\sin \varphi_{i} \approx \varphi_{i}, \cos \varphi_{i} \approx 1$. We define

$$
\begin{aligned}
\bar{r}_{i} & =r_{i}-c \dot{s}_{i}, \\
h_{i} & =\frac{1}{1-c d_{i}}, \\
\tau_{3 i} & =\frac{1}{m_{u}}\left(\tau_{u i}-d_{u} u_{i}\right), \\
\tau_{4 i} & =\frac{1}{m_{r}}\left(\tau_{r i}-d_{r} r_{i}\right)-c h_{i} \tau_{3 i} .
\end{aligned}
$$

Then, the dynamics of vehicle $i$ can be decoupled into two subsystems: the surge subsystem

$$
\begin{aligned}
& \dot{s}_{i}=h_{i} u_{i}, \\
& \dot{u}_{i}=\tau_{3 i},
\end{aligned}
$$

and the yaw subsystem 


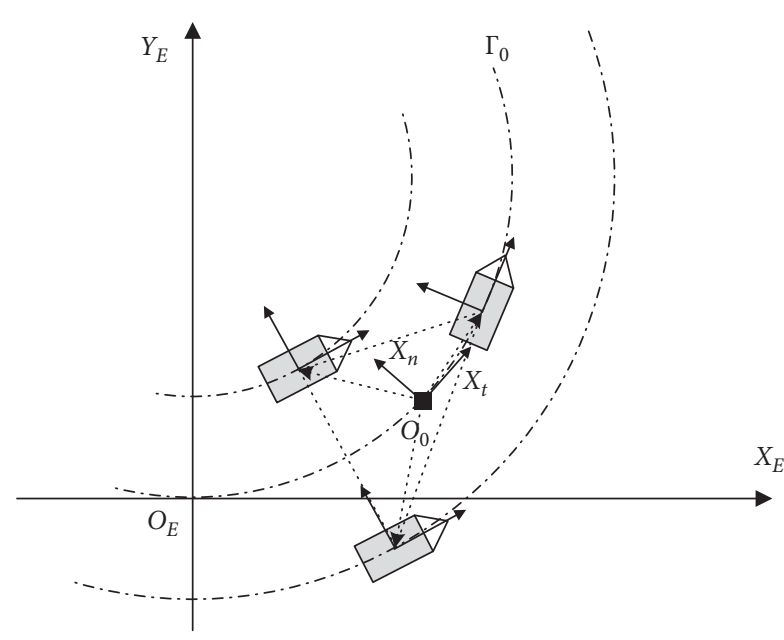

Figure 3: The virtual leader and the Serret-Frenet frame.

$$
\begin{aligned}
\dot{d}_{i} & =u_{i} \varphi_{i}, \\
\dot{\varphi}_{i} & =\bar{r}_{i}, \\
\dot{\bar{r}}_{i} & =\tau_{4 i} .
\end{aligned}
$$

Suppose that $h_{i}, i=1, \ldots m$ are not zero. The cascade subsystem (29) can be written as

$$
\begin{aligned}
\dot{s}_{i} & =u_{1 i}^{\prime}, \\
\dot{u}_{1 i}^{\prime} & =\tau_{3 i}^{\prime},
\end{aligned}
$$

where $u_{i}^{\prime}=h_{i} u_{i}$ and $\tau_{3 i}^{\prime}=h_{i} \tau_{3 i}$. The finite-time distributed protocols based on the relative position and relative velocity are designed as

$$
\begin{aligned}
\tau_{3 i}^{\prime}= & -k_{5 i}\left(\sum_{j=1}^{m} a_{i j}\left(s_{i}-s_{j}-\left(\Delta s_{i}-\Delta s_{j}\right)\right)+a_{i 0}\left(\left(s_{i}-\Delta s_{i}\right)-s_{0}\right)\right)^{\alpha_{5}} \\
& -k_{6 i}\left(\sum_{j=0}^{m} a_{i j}\left(u_{i}^{\prime}-u_{j}^{\prime}\right)+a_{i 0}\left(u_{i}^{\prime}-u_{0}^{\prime}\right)\right)^{\alpha_{6}}, \quad \text { for } t \in\left(t_{f 1}, \infty\right),
\end{aligned}
$$

where the exponents $\alpha_{5}$ and $\alpha_{6}$ are selected as $\alpha_{5}=\alpha_{6} /\left(2-\alpha_{6}\right), 0<\alpha_{6}<1$. The gains $k_{5 i}$ and $k_{6 i}$ are selected such that $s^{2}+k_{6 i} s+k_{5 i}$ are Hurwitz polynomials.

Similarly, $u_{i}, i=1, \ldots m$ are assumed to change slowly and not zero. The cascade system (30) can be written as

$$
\begin{gathered}
\dot{d}_{i}=\varphi_{i}^{\prime}, \\
\dot{\varphi}_{i}^{\prime}=\vec{r}_{i}^{\prime}, \\
\dot{\bar{r}}_{i}^{\prime}=\tau_{4 i}^{\prime},
\end{gathered}
$$

where $\varphi_{i}^{\prime}=u_{i} \varphi^{\prime}, \bar{r}_{i}^{\prime}=u_{i} \bar{r}_{i}$, and $\tau_{4 i}^{\prime}=u_{i} \tau_{4 i}$. The finite-time distributed protocols based on the relative position and relative velocity are designed as

$$
\begin{aligned}
\tau_{4 i}^{\prime}= & -k_{7 i}\left(\sum_{j=1}^{m} a_{i j}\left(d_{i}-d_{j}-\left(\Delta d_{i}-\Delta d_{j}\right)\right)+a_{i 0}\left(\left(d_{i}-\Delta d_{i}\right)-d_{0}\right)\right)^{\alpha_{7}} \\
& -k_{8 i}\left(\sum_{j=1}^{m} a_{i j}\left(\varphi_{i}^{\prime}-\varphi_{j}^{\prime}\right)+a_{i 0}\left(\varphi_{i}^{\prime}-\varphi_{0}^{\prime}\right)\right)^{\alpha_{8}} \\
& -k_{9 i}\left(\sum_{j=1}^{m} a_{i j}\left(\bar{r}_{i}^{\prime}-\bar{r}_{j}^{\prime}\right)+a_{i 0}\left(\bar{r}_{i}^{\prime}-\bar{r}_{0}^{\prime}\right)\right)^{\alpha_{9}}, \quad \text { for } t \in\left(t_{f 1}, \infty\right),
\end{aligned}
$$

where $\alpha_{7}, \alpha_{8}$, and $\alpha_{9}$ are selected as $\alpha_{7}=\alpha_{8} \alpha_{9} /$ $\left(2 \alpha_{9}-\alpha_{8}\right)$ and $\alpha_{8}=\alpha_{9} /\left(2-\alpha_{9}\right), 0<\alpha_{9}<1$. The gains $k_{7 i}, k_{8 i}$, and $k_{9 i}$ are selected such that $s^{3}+k_{9 i} s^{2}+k_{8 i} s+k_{7 i}$ are Hurwitz polynomials.

The main result is given by the following theorem.

Theorem 1. Under Assumption 1, protocols (32) and (34) can guide system (31) and (33) to realize finite-time consensus, respectively.

Proof. For systems (33) and (34), define the error states as

$$
\begin{aligned}
& e_{i, 1}=\sum_{j=1}^{m} a_{i j}\left(d_{i}-d_{j}-\left(\Delta d_{i}-\Delta d_{j}\right)\right)+a_{i 0}\left(\left(d_{i}-\Delta d_{i}\right)-d_{0}\right), \\
& e_{i, 2}=\sum_{j=1}^{m} a_{i j}\left(\varphi_{i}^{\prime}-\varphi_{j}^{\prime}\right)+a_{i 0}\left(\varphi_{i}^{\prime}-\varphi_{0}^{\prime}\right), \\
& e_{i, 3}=\sum_{j=1}^{m} a_{i j}\left(\bar{r}_{i}^{\prime}-\bar{r}_{j}^{\prime}\right)+a_{i 0}\left(\bar{r}_{i}^{\prime}-\bar{r}_{0}^{\prime}\right) .
\end{aligned}
$$

Denoting $\quad \mathbf{e}_{1}=\left(e_{1,1}, e_{2,1}, \ldots e_{m, 1}\right)^{T}, \mathbf{e}_{2}=\left(e_{1,2}, e_{2,2}\right.$, $\left.\ldots e_{m, 2}\right)^{T}$, and $\mathbf{e}_{3}=\left(e_{1,3}, e_{2,3}, \ldots e_{m, 3}\right)^{T}$, it is straightforward to express the error dynamics as

$$
\begin{aligned}
& \dot{e}_{1}=\mathbf{e}_{2}, \\
& \dot{e}_{2}=\mathbf{e}_{3}, \\
& \dot{e}_{3}=(\mathbf{L}+\mathbf{B})\left(-\mathbf{K}_{7} \mathbf{e}_{1}^{a_{7}}-\mathbf{K}_{8} \mathbf{e}_{2}^{a_{8}}-\mathbf{K}_{9} \mathbf{e}_{3}^{a_{9}}\right),
\end{aligned}
$$

where $\mathbf{K}_{7}=\operatorname{diag}\left\{k_{71}, k_{72}, \ldots, k_{7 m}\right\}, \mathbf{K}_{8}=\operatorname{diag}\left\{k_{81}, k_{82}, \ldots\right.$, $\left.k_{8 m}\right\}, \mathbf{K}_{9}=\operatorname{diag}\left\{k_{91}, k_{92}, \ldots, k_{9 m}\right\}$, and $\mathbf{e}_{j}^{\alpha}, j=1,2,3$, denotes $\left(e_{1, j}^{\alpha}, e_{2, j}^{\alpha}, \ldots, e_{m, j}^{\alpha}\right)^{T}$.

$$
\text { Let }
$$

$$
\begin{aligned}
& \mathbf{A}_{e}=\left(\begin{array}{ccc}
0 & \mathbf{I} & 0 \\
0 & 0 & \mathbf{I} \\
-\mathbf{K}_{7} & -\mathbf{K}_{8} & -\mathbf{K}_{9}
\end{array}\right) \\
& \mathbf{M}=\left(\begin{array}{lll}
\mathbf{I} & & \\
& \mathbf{I} & \\
& & \mathbf{L}+\mathbf{B}
\end{array}\right), \\
& \overline{\mathbf{A}}_{e}=\mathbf{M \mathbf { A } _ { e }}=\left(\begin{array}{ccc}
0 & \mathbf{I} & 0 \\
0 & 0 & \mathbf{I} \\
-(\mathbf{L}+\mathbf{B}) \mathbf{K}_{7} & -(\mathbf{L}+\mathbf{B}) \mathbf{K}_{8} & -(\mathbf{L}+\mathbf{B}) \mathbf{K}_{9}
\end{array}\right) \text {. }
\end{aligned}
$$


Consider a Lyapunov function candidate $\mathbf{V}(\mathbf{e})=\mathbf{e}^{T} \mathbf{P}_{e} \mathbf{e}$, where $\mathbf{e}=\left(\mathbf{e}_{1}^{T}, \mathbf{e}_{2}^{T}, \mathbf{e}_{3}^{T}\right)^{T}$ and $\mathbf{P}_{e}=\mathbf{P}_{e}^{T}>0$. It is obviously that $\mathbf{A}_{e}$ is a Hurwitz satisfying the Lyapunov equation $\mathbf{P}_{e} \mathbf{A}_{e}+\mathbf{A}_{e}^{T} \mathbf{P}_{e}=-\mathbf{Q}_{e}$, where $\mathbf{Q}_{e}=\mathbf{Q}_{e}^{T}>0$. Since $\mathbf{M}$ is a symmetric positive definite matrix, the Lyapunov equation $\overline{\mathbf{P}}_{e} \overline{\mathbf{A}}_{e}+\overline{\mathbf{A}}_{e}^{T} \overline{\mathbf{P}}_{e}=-\mathbf{Q}_{e}$ holds with $\overline{\mathbf{P}}_{e}=\mathbf{P}_{e} \mathbf{M}^{-1}>0$, which means that $\bar{V}(\mathbf{e})=\mathbf{e}^{T} \overline{\mathbf{P}}_{e} \mathbf{e}$ is a Lyapunov function for the linear system $\dot{e}=\bar{A}_{e} \mathbf{e}$.

From the proof of Theorem 1 in [33], we can see that $V\left(\zeta_{e}\right)=\zeta_{e}^{T} \overline{\mathbf{P}}_{e} \zeta_{e}$ is a Lyapunov function for system (36), where $\zeta_{e}=\left(\left(\mathbf{e}_{1}^{\alpha_{7}}\right)^{T},\left(\mathbf{e}_{2}^{\alpha_{8}}\right)^{T},\left(\mathbf{e}_{3}^{\alpha_{9}}\right)^{T}\right)^{T}$. So, system (36) is asymptotically stable. Moreover, it can be verified that system (36) is a homogeneous system of degree $\alpha_{9}-1 / \alpha_{9}<0$. Hence, by Lemma 2, system (36) is finite-time convergent to the origin. It follows that the finite-time consensus problem of system (33) with protocol (34) is solved.

The finite-time consensus of system (31) with protocol (32) can be proved similarly. Theorem 1 is proven.

\section{Numerical Simulation and Results}

To illustrate the effectiveness of the proposed control law, a simulation study based on the model is presented in [15]. The same model parameters are chosen as in [38] and given as follows: $m_{u}=25.8, m_{v}=33.8, m_{r}=2.76, m_{u v}=6.2$, $d_{u}=12+2.5|u|, d_{v}=17+4.5|v|$, and $d_{r}=0.5+0.1|r|$.

Without loss of generality, in our simulation scene, we assume that there are only 3 USVs in the formation. Consider the fact that not all the follower vehicles can receive information from each other, but all of them can directly communicate with the "virtual leader," as depicted in Figure 4. In addition, their initial conditions and the desired positions and orientations of each USV in the first stage are listed in Table 1.

According to the setting of aforementioned scenario, the adjacent matrix $\mathbf{A}$, the degree matrix $\mathbf{D}$, the Laplace matrix $\mathbf{L}$, and the leader adjacency matrix $\mathbf{B}$ can be deduced as follows:

$$
\begin{aligned}
\mathbf{A} & =\left[\begin{array}{lll}
0 & 1 & 0 \\
1 & 0 & 1 \\
0 & 1 & 0
\end{array}\right], \\
\mathbf{D} & =\left[\begin{array}{lll}
1 & 0 & 0 \\
0 & 2 & 0 \\
0 & 0 & 1
\end{array}\right], \\
\mathbf{L} & =\left[\begin{array}{ccc}
1 & -1 & 0 \\
-1 & 2 & -1 \\
0 & -1 & 1
\end{array}\right], \\
\mathbf{B} & =\left[\begin{array}{lll}
1 & 0 & 0 \\
0 & 1 & 0 \\
0 & 0 & 1
\end{array}\right] .
\end{aligned}
$$

The expected path of the group centroid is given as a circle with radius $100 \mathrm{~m}$. Thus, the expected paths for USV1, USV2, and USV3 are concentric circles with radius $90 \mathrm{~m}, 100 \mathrm{~m}$, and $110 \mathrm{~m}$, respectively. According to the design guideline, the control parameters are chosen as listed in Tables 2 and 3.

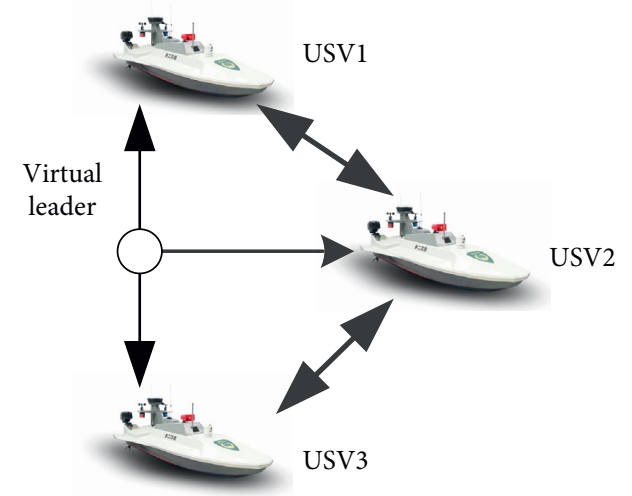

FIgURE 4: The communication links relationship in the formation.

TABLE 1: Initial and desired states of the USVs.

\begin{tabular}{lc}
\hline \multirow{3}{*}{ Initial states } & USV1: $[-30,60, \pi / 3,0,0,-\pi / 18]$ \\
& USV2: $[-20,-30, \pi / 2,0,0,-\pi / 18]$ \\
& USV3: $[50,-50,-\pi / 4,0,0, \pi / 18]$ \\
\hline \multirow{3}{*}{ Desired states in formation } & USV1: $[0,10,0,0,0,0]$ \\
& USV2: $[6.3,0.2, \pi / 50,0,0,0]$ \\
& USV3: $[0,-10,0,0,0,0]$ \\
\hline
\end{tabular}

TABLE 2: The gains of the controllers.

\begin{tabular}{lccccccccc}
\hline Gains & $k_{1}$ & $k_{2}$ & $k_{3}$ & $k_{4}$ & $k_{5}$ & $k_{6}$ & $k_{7}$ & $k_{8}$ & $k_{9}$ \\
\hline USV1 & 5 & 0.5 & 0.1 & 1 & 0.5 & 0.5 & 5 & 5 & 5 \\
USV2 & 5 & 0.5 & 0.1 & 1 & 0.5 & 0.5 & 5 & 5 & 5 \\
USV3 & 5 & 0.5 & 0.1 & 1 & 0.5 & 0.5 & 5 & 5 & 5 \\
\hline
\end{tabular}

TABle 3: The exponents of the controllers.

\begin{tabular}{lccccccccc}
\hline Exponents & $\alpha_{1}$ & $\alpha_{2}$ & $\alpha_{3}$ & $\alpha_{4}$ & $\alpha_{5}$ & $\alpha_{6}$ & $\alpha_{7}$ & $\alpha_{8}$ & $\alpha_{9}$ \\
\hline USV & 1.81 & 0.90 & 1.81 & 0.90 & 0.20 & 0.33 & 0.25 & 0.33 & 0.50 \\
\hline
\end{tabular}

The final convergence time $t_{f 1}$ of the first stage for all USVs is selected as $150 \mathrm{~s}$, which is more than the sum of $t_{1 i}$, $t_{2 i}$, and $t_{3 i}$. In the second stage, we assume that there is a virtual leader moving along the path with a fixed velocity $u_{t}=2 \mathrm{~m} / \mathrm{s}$.

The simulation results are shown in Figures 5-8. Figure 5 shows the formation trajectory of the USVs under the proposed control scheme. It can be seen that each of the USV s could arrive at their own starting points in formation first and then could track the desired paths and maintain the triangular pattern well.

Figure 6 shows the curves of the states and control inputs of the USVs. It can be observed that, in the first stage, the positions of the USVs converge to the expected values within about 40 seconds, before the first switch of the control algorithm. And then the orientations of the USV converge to the expected values within about 140 seconds, which is also before the second switching time of the 


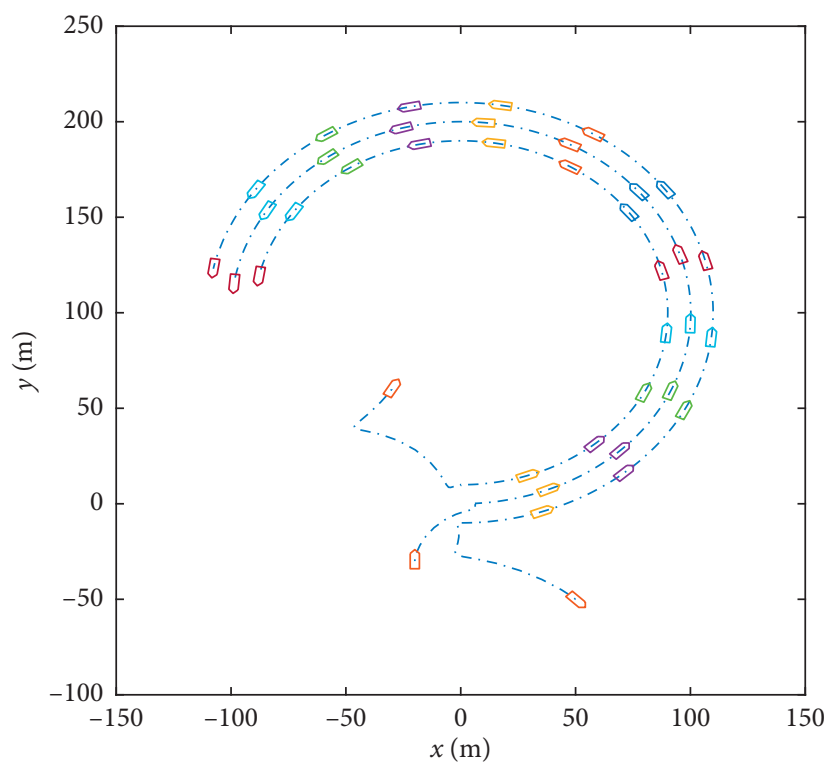

FIgURE 5: Trajectory of the USVs.

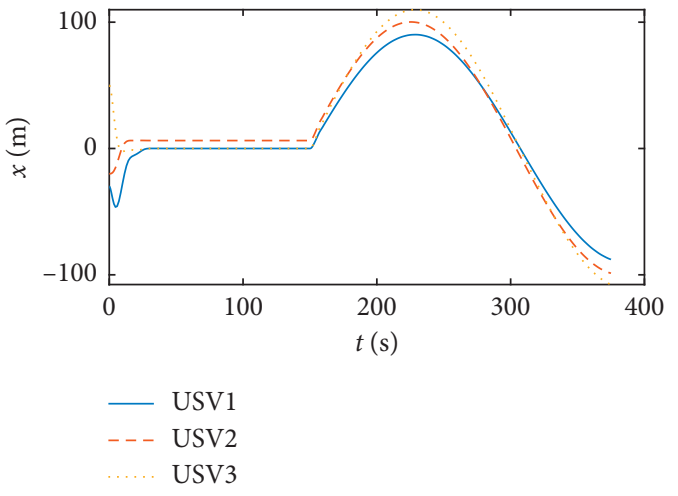

(a)

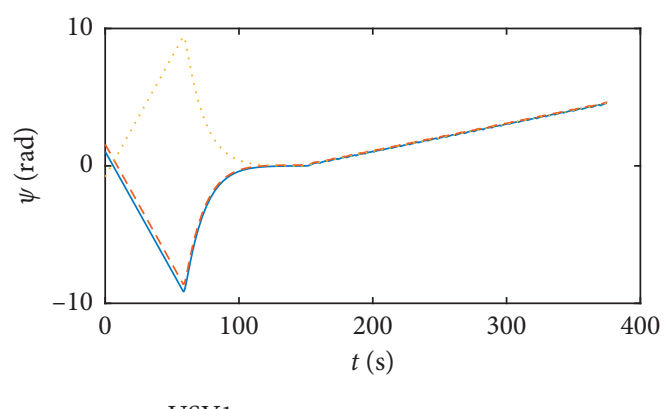

- USV1

- - - USV2

USV3

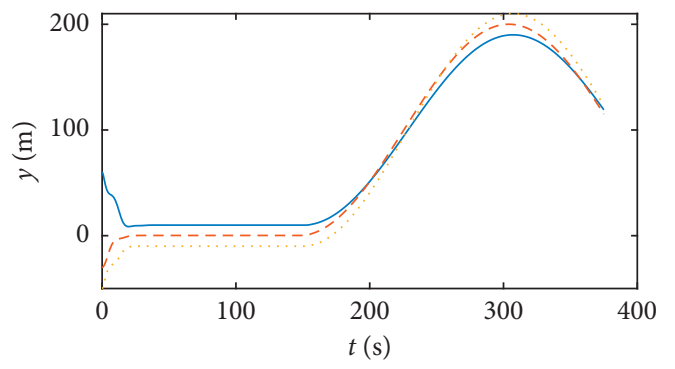

— USV1

- - - USV2

USV3

(b)

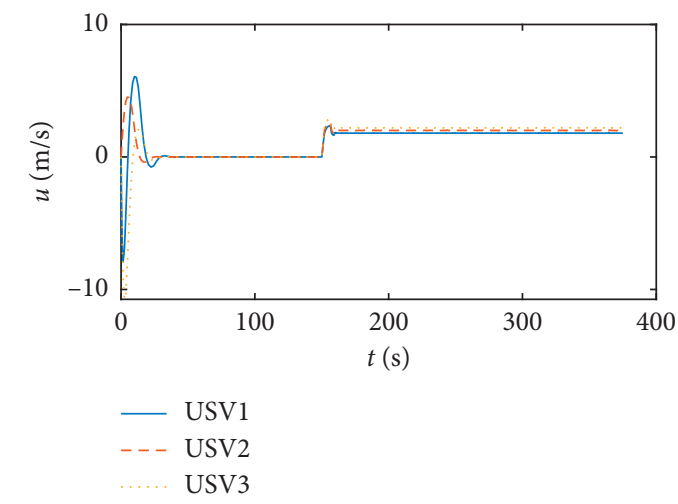

(d)

Figure 6: Continued. 


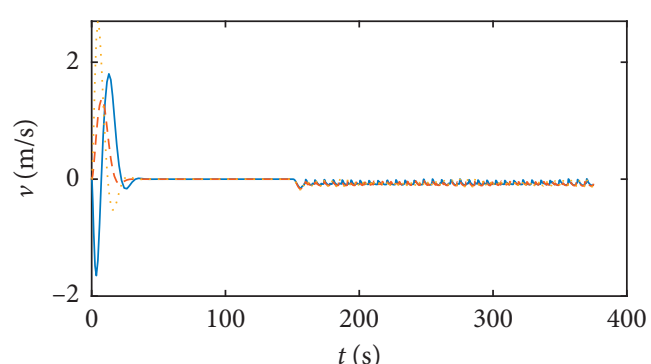

USV 1

- - - USV2

USV3

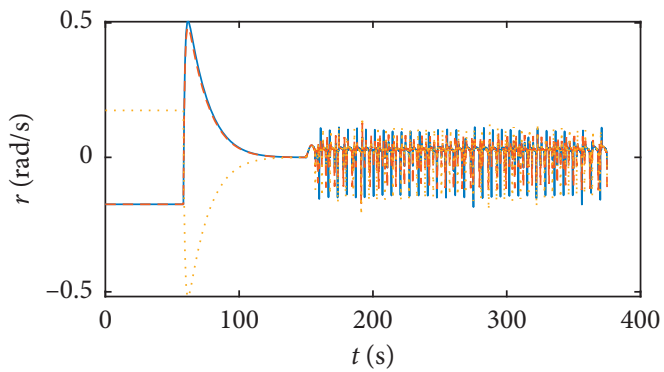

- USV1

- - - USV2

USV3

(e)

(f)

FIgURE 6: Curves of states with respect to time.
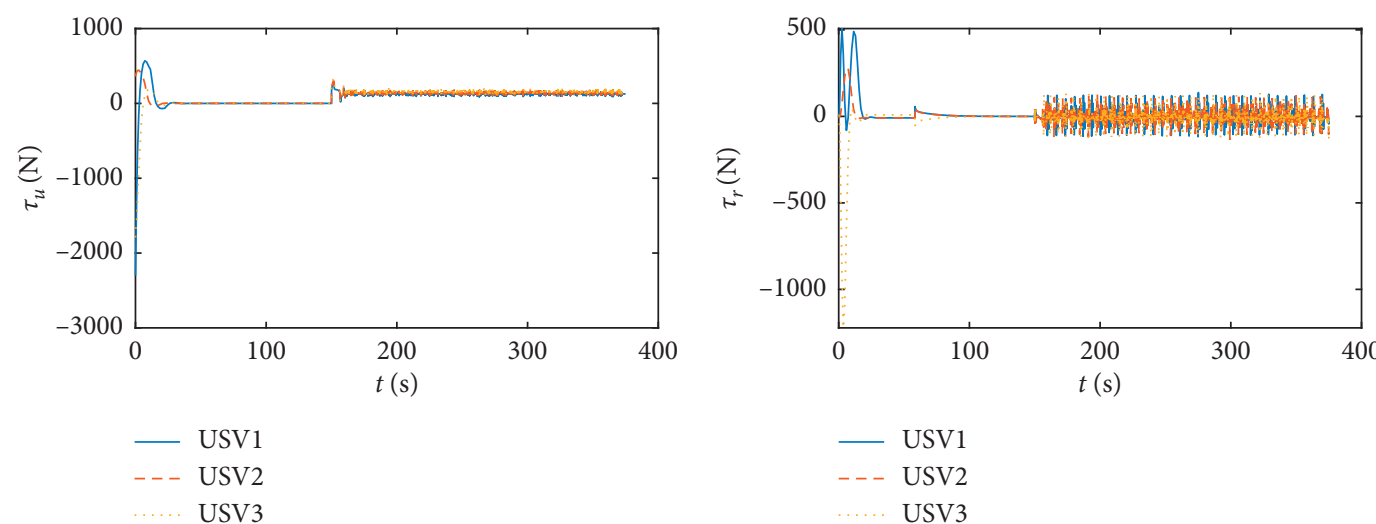

(a)

(b)

Figure 7: Curves of control efforts with respect to time.

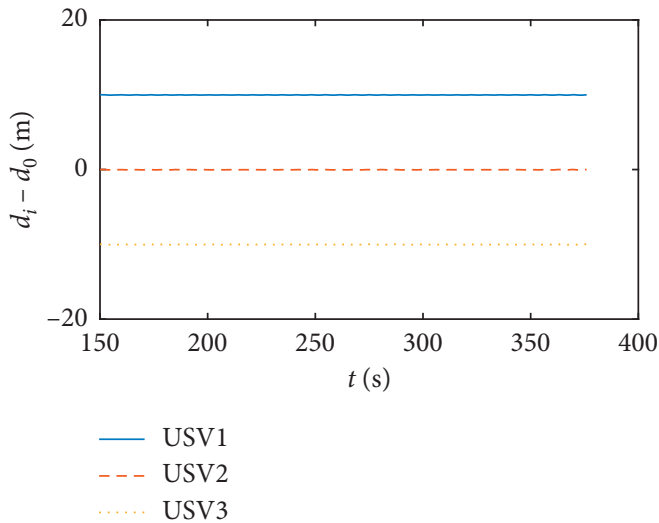

(a)

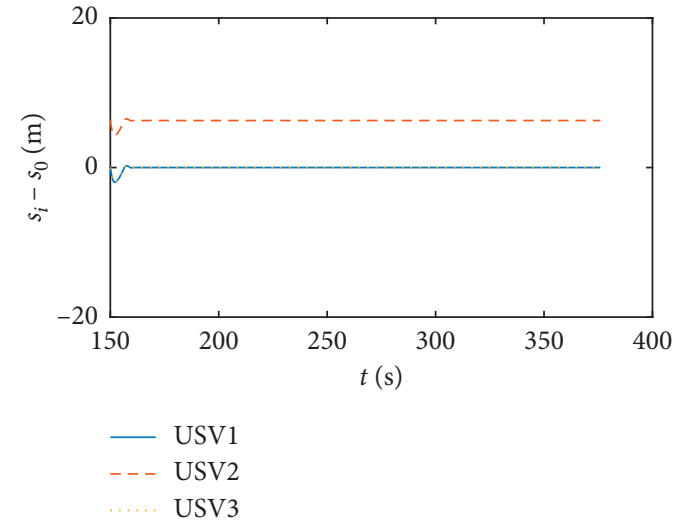

(b)

Figure 8: Curves of state errors $d_{i}-d_{0}$ and $s_{i}-s_{0}$ with respect to time.

control algorithm. The control inputs of the USVs are depicted in Figure 7. In the first stage, the exponents $\alpha_{2}$ and $\alpha_{4}$ have great influences on the convergence time. A good choice of them can greatly reduce the settling time of stage one. In the second stage, the feedback gains of the yaw subsystem $k_{7 i}, k_{8 i}$, and $k_{9 i}$ are usually selected to more than the gains of the surge subsystem $k_{5 i}$ and $k_{6 i}$, to make the USVs on their paths. 
Figure 8 shows the errors of the longitudinal displacement and the transversal displacement between the follower vehicles and the leader vehicle. The cooperative control starts working after which every USV gets to its own start points. Therefore, the time starts at 150 seconds and the errors of the displacements are constant, which means that the formation of the USV is kept well.

\section{Conclusion}

A formation control strategy based on finite-time control and leader-follower theory is proposed in the paper to achieve formation maneuver control for USVs. The formation control process is divided into two stages. In the first stage, all the USVs would be steered to their starting points in the formation pattern in finite time. Then, in the second stage, all the vehicles would be controlled to maintain the formation pattern based on finite-time distributed cooperative control. By using tools from homogeneous theory, it is theoretically shown that the proposed protocol can guarantee finite-time converge and consensus, respectively. Numerical simulation is made to illustrate effectiveness of our strategy.

\section{Data Availability}

The data used to support the findings of this study are available from the corresponding author upon request.

\section{Conflicts of Interest}

The authors declare no conflicts of interest.

\section{Authors' Contributions}

X. Y. and H. Y. conceptualized the study; H. Y. and X. Y. developed methodology; C. G. and H. Y. developed software; C. G. and Z. D. carried out formal analysis; C. G. investigated the study; H. Y, C. G., and X. Y. wrote the original draft.

\section{Acknowledgments}

This work was supported in part by the National Natural Science Foundation of China under Grant 61903163, Natural Science Foundation of the Jiangsu Higher Education Institutions of China under Grants 18KJB520010 and 19KJB510023, Jiangsu Province Postgraduate Innovation Plan under Grant 1032161916, and Zhenjiang Key Research and Design Project under Grant GY2018026.

\section{References}

[1] Z. Liu, Y. Zhang, X. Yu, and C. Yuan, "Unmanned surface vehicles: an overview of developments and challenges," $A n$ nual Reviews in Control, vol. 41, pp. 71-93, 2016.

[2] Z. Dong, L. Wan, Y. Li, T. Liu, and G. Zhang, "Trajectory tracking control of underactuated USV based on modified backstepping approach," International Journal of Naval Architecture and Ocean Engineering, vol. 7, no. 5, pp. 817-832, 2015.

[3] Y.-L. Liao, M.-J. Zhang, L. Wan, and Y. Li, "Trajectory tracking control for underactuated unmanned surface vehicles with dynamic uncertainties," Journal of Central South University, vol. 23, no. 2, pp. 370-378, 2016.

[4] Y.-L. Liao, M.-J. Zhang, and L. Wan, "Serret-frenet frame based on path following control for underactuated unmanned surface vehicles with dynamic uncertainties," Journal of Central South University, vol. 22, no. 1, pp. 214-223, 2015.

[5] T. Liu, Z. Dong, H. Du, L. Song, and Y. Mao, "Path following control of the underactuated USV based on the improved line-of-sight guidance algorithm," Polish Maritime Research, vol. 24, no. 1, pp. 3-11, 2017.

[6] K.-C. Cao and Y.-P. Tian, "A time-varying cascaded design for trajectory tracking control of non-holonomic systems," International Journal of Control, vol. 80, no. 3, pp. 416-429, 2007.

[7] Z. C. Zhang, Stabilization and Tracking Control for Nonholonomic and Underactuated System, Ph.D. thesis, Southeast University of China, Nanjing, China, 2016.

[8] B. W. Zeng, Research on Nonlinear Control of Waterjet Propulsion Surface Unmanned Naval Vessel, Ph.D. thesis, Harbin Engineering University, Harbin, China, 2012.

[9] R. W. Beard, J. Lawton, and F. Y. Hadaegh, "A coordination architecture for spacecraft formation control," IEEE Transactions on Control Systems Technology, vol. 9, no. 6, pp. 777-790, 2001.

[10] X. Cai and M. D. Queiroz, "Adaptive rigidity-based formation control for multirobotic vehicles with dynamics," IEEE Transactions on Control Systems Technology, vol. 23, no. 1, pp. 389-396, 2015.

[11] T. Balch and R. C. Arkin, "Behavior-based formation control for multirobot teams," IEEE Transactions on Robotics and Automation, vol. 14, no. 6, pp. 926-939, 1998.

[12] L. Consolini, F. Morbidi, D. Prattichizzo, and M. Tosques, "Leader-follower formation control of nonholonomic mobile robots with input constraints," Automatica, vol. 44, no. 5, pp. 1343-1349, 2008.

[13] K. D. Godard, K. Dev Kumar, and A. Zou, "Robust stationkeeping and reconfiguration of underactuated spacecraft formations," Acta Astronautica, vol. 105, no. 2, pp. 495-510, 2014.

[14] J. Wang, J. Y. Liu, and H. Yi, "Formation control of unmanned surface vehicles with vision sensor constraints," 2015.

[15] Z. H. Peng and D. Wang, "Adaptive dynamic surface control for formations of autonomous surface vehicles with uncertain dynamics," IEEE Transactions on Control Systems Technology, vol. 21, no. 2, pp. 513-520.

[16] Z. Sun, G. Zhang, Y. Lu, and W. Zhang, "Leader-follower formation control of underactuated surface vehicles based on sliding mode control and parameter estimation," ISA Transactions, vol. 72, pp. 15-24, 2018.

[17] K. Shojaei, "Leader-follower formation control of underactuated autonomous marine surface vehicles with limited torque," Ocean Engineering, vol. 105, pp. 196-205, 2015.

[18] H. Li, P. Xie, and W. Yan, "Receding horizon formation tracking control of constrained underactuated autonomous underwater vehicles," IEEE Transactions on Industrial Electronics, vol. 64, no. 6, pp. 5004-5013, 2017.

[19] J. Li, J. Du, and W.-J. Chang, "Robust time-varying formation control for underactuated autonomous underwater vehicles with disturbances under input saturation," Ocean Engineering, vol. 179, pp. 180-188, 2019.

[20] X. Jin, "Fault tolerant finite-time leader-follower formation control for autonomous surface vessels with LOS range and angle constraints," Automatica, vol. 68, pp. 228-236, 2016. 
[21] W. Dong, "Cooperative control of underactuated surface vessels," IET Control Theory \& Applications, vol. 4, no. 9, pp. 1569-1580, 2010.

[22] Z. H. Peng and J. Wang, "Distributed maneuvering of autonomous surface vehicles based on neurodynamic optimization and fuzzy approximation," IEEE Trans. on Control Systems Technology, vol. 26, no. 3, pp. 1083-1090, 2017.

[23] L. Liu, D. Wang, Z. Peng, and T. Li, "Modular adaptive control for LOS-based cooperative path maneuvering of multiple underactuated autonomous surface vehicles," IEEE Transactions on Systems, Man, and Cybernetics: Systems, vol. 47, no. 7, pp. 1613-1624, 2017.

[24] N. Wang and C. J. Qian, "Adaptive robust finite-time trajectory tracking control of fully actuated marine surface vehicles," IEEE Transactions on Control Systems Technology, vol. 24, no. 4, pp. 1454-1462, 2015.

[25] N. Wang and Z. Sun, "Finite-time observer based guidance and control of underactuated surface vehicles with unknown sideslip angles and disturbances," IEEE Access, vol. 6, no. 6, pp. 14060-14070, 2018.

[26] Q. Hou, L. Ma, S. Ding, X. Yang, and X. Chen, "Composite finite-time straight-line path-following control of an underactuated surface vessel," Journal of the Franklin Institute, vol. 357, no. 16, Article ID 11496, 2020.

[27] M. Ghasemi and S. G. Nersesov, "Finite-time coordination in multiagent systems using sliding mode control approach," Automatica, vol. 50, no. 4, pp. 1209-1216, 2014.

[28] Y. Liu and Z. Geng, "Finite-time formation control for linear multi-agent systems: a motion planning approach," Systems \& Control Letters, vol. 85, pp. 54-60, 2015.

[29] Y. Zhao, Z. Duan, and G. Wen, "Finite-time consensus for second-order multi-agent systems with saturated control protocols," IET Control Theory \& Applications, vol. 9, no. 3, pp. 312-319, 2014.

[30] C. Huang, X. Zhang, and G. Zhang, "Improved decentralized finite-time formation control of underactuated USVs via a novel disturbance observer," Ocean Engineering, vol. 174, pp. 117-124, 2019.

[31] T. Li, R. Zhao, C. L. P. Chen, L. Fang, and C. Liu, "Finite-time formation control of under-actuated ships using nonlinear sliding mode control," IEEE Transactions on Cybernetics, vol. 48, no. 11, pp. 3243-3253, 2018.

[32] S. H. Ding and S. H. Li, "A survey for finite-time control problems," Control and Decision, vol. 26, no. 2, pp. 161-169, 2011.

[33] M. Basin, Y. Shtessel, and F. Aldukali, "Continuous finite- and fixed-time high-order regulators," Journal of the Franklin Institute, vol. 353, no. 18, pp. 5001-5012, 2016.

[34] C. Godsil and G. F. Royle, Algebraic Graph Theory, Springer Science \& Business Media, New York, NY, USA, 2013.

[35] F. L. Lewis, H. Zhang, K. Hengster-Movric et al., Cooperative Control of Multi-Agent Systems: Optimal and Adaptive Design Approaches, Springer Science \& Business Media, New York City, NY, USA, 2013.

[36] J. Cheng, J. Yi, and D. Zhao, "Stabilization of an underactuated surface vessel via discontinuous control," in Proceedings of the American Control Conference, pp. 206-211, Denver, CO, USA, 2007.

[37] K. D. Do and J. Pan, "State-and output-feedback robust pathfollowing controllers for underactuated ships using serretfrenet frame," Ocean Engineering, vol. 31, no. 5, pp. 587-613, 2004.
[38] K. D. Do and J. Pan, "Global robust adaptive path following of underactuated ships," Automatica, vol. 42, no. 10, pp. 1713-1722, Oct. 2006. 